\title{
Distal end of the atrioventricular nodal artery predicts the risk of atrioventricular block during slow pathway catheter ablation of atrioventricular nodal re-entrant tachycardia
}

\author{
J-L Lin, S K S Huang, L-P Lai, L-J Lin, J-H Chen, Y-Z Tseng, W-P Lien
}

\begin{abstract}
Objective-To search for a reliable anatomical landmark within Koch's triangle to predict the risk of atrioventricular (AV) block during radiofrequency slow pathway catheter ablation of AV nodal re-entrant tachycardia (AVNRT).

Patients and methods-To test the hypothesis that the distal end of the AV nodal artery represents the anatomical location of the AV node, and thus could be a useful landmark for predicting the risk of AV block, 128 consecutive patients with AVNRT receiving slow pathway catheter ablation were prospectively studied in two phases. In phase I (77 patients), angiographic demonstration of the AV nodal artery and its ending was performed at the end of the ablation procedure, whereas in the subsequent phase II study (51 patients), the angiography was performed immediately before catheter ablation to assess the value of identifying this new landmark in reducing the risk of AV block. Multiple electrophysiologic and anatomical parameters were analysed. The former included the atrial activation sequence between the His bundle recording site (HBE) and the coronary sinus orifice or the catheter ablation site, either during AVNRT or during sinus rhythm. The latter included the spatial distances between the distal end of the AV nodal artery and the HBE and the final catheter ablation site, and the distance between the HBE and the tricuspid border at the coronary sinus orifice floor.

Results-In phase I, nine of the 77 patients had complications of transient (seven patients) or permanent (two patients) complete AV block during stepwise, anatomy guided slow pathway catheter ablation. These nine patients had a wider distance between the HBE and the distal end of the AV nodal artery, and a closer approximation of the catheter ablation site to the distal end of the AV nodal artery, which independently predicted the risk of AV block. In contrast, none of the available electrophysiologic parameters were shown to be reliable. When the distance between the distal end of the AV nodal artery and the ablation target site was more than $2 \mathrm{~mm}$, the complication of AV block virtually never occurred. In phase II, all 51 patients had successful elimination of the slow pathways without complication when the ablation procedure was guided by preceding angiography with identification of the distal end of the AV nodal artery.

Conclusions-The distal end of the AV nodal artery shown by angiography serves as a useful landmark for the prediction of the risk of AV block during slow pathway catheter ablation of AVNRT.

(Heart 2000;83:543-550)
\end{abstract}

Division of Cardiology, Department of Internal Medicine, National Taiwan University Hospital, Taipei, Taiwan $\mathrm{J}-\mathrm{L}$ Lin

S K S Huang

L-P Lai

Y-Z Tseng

W-P Lien

Division of Cardiology,

Department of

Internal Medicine,

National Cheng-Kung

University Hospital,

Tainan, Taiwan

L-J Lin

$\mathrm{J}-\mathrm{H}$ Chen

Correspondence to: Dr S K S Huang,

Department of Internal

Medicine, National Taiwan

University College of

Medicine, No1, Section 1,

Jen-Ai Road, Room 208,

Taipei 10018, Taiwan

email: SKH@

ha.mc.ntu.edu.tw

Accepted 7 January 2000

Keywords: atrioventricular nodal artery; atrioventricular nodal re-entrant tachycardia; catheter ablation; heart block.

It is generally believed that the dual atrioventricular (AV) nodal pathways of AV nodal re-entrant tachycardia (AVNRT) are two functionally and anatomically distinct structures, in which the fast pathway appears to be the anterior-superior atrionodal connections near the AV node and the His bundle, while the slow pathway appears to be the posterior-inferior atrionodal connections near the coronary sinus orifice. ${ }^{12}$ Radiofrequency (RF) catheter ablation of the slow pathway has been proved to be effective in the treatment of recurrent AVNRT..$^{3-5}$ Inadvertent complication of permanent complete AV block is rare. ${ }^{5}{ }^{6}$ However, the incidence of transient complete AV block during slow pathway catheter ablation remains significant, ranging from $2-24 \% .^{7-11}$ Unless the delivery of RF energy is immediately interrupted, the risk of permanent damage to normal AV conduction is very high. ${ }^{8-11}$ Several electrophysiological parameters harbingering the potential risk of AV block during slow pathway catheter ablation of AVNRT were proposed. These warning electrical signals include the retrograde atrial activation time difference between the His bundle recording site and the coronary sinus orifice in AVNRT, ${ }^{4}$ the antegrade atrial activation time difference between the His bundle recording site and the catheter ablation site in sinus rhythm, ${ }^{12}$ junctional tachycardia cycle length during RF energy application, ${ }^{13}$ and junctional ectopies with retrograde ventriculoatrial (VA) block. ${ }^{9}{ }^{12}{ }^{13}$ Unfortunately, none of these signals could be used to serve as a powerful predictor to avoid the occurrence of AV block because of inadequate predictive accuracy. ${ }^{9}{ }^{12} 13$ A reliable landmark is still lacking.

The AV nodal artery is the main artery supplying the AV node and sometimes the proximal His bundle. ${ }^{14}{ }^{15}$ Selective catheterisation and application of pharmacological agents 
including ethanol has been shown to damage specifically AV nodal function. ${ }^{16-18}$ Thus, we hypothesised that the distal end of the AV nodal artery visualised by angiography could be a useful landmark for the anatomical location of the AV node within Koch's triangle. The approximation of the tip of the ablation catheter to the distal end of the AV nodal artery would carry the highest risk of complete AV block in slow pathway catheter ablation.

In order to test the hypothesis, we conducted a study in two separate phases. In phase I, selective coronary angiography to demonstrate the AV nodal artery and its ending was performed at the end of the slow pathway catheter ablation. This would allow us to find the relation between the risk of AV block and the location of the distal end of the AV nodal artery and the ablation target site. In the subsequent phase II study, the distal end of the AV nodal artery was demonstrated by angiography before slow pathway catheter ablation. Thus, the applicability and usefulness of identifying this anatomical landmark in reducing the risk of AV block can be further evaluated.

\section{Methods}

PATIENT POPULATION

Seventy seven consecutive patients undergoing RF catheter ablation for proven AVNRT were prospectively enrolled between October 1996 and September 1997 for phase I of the study. There were 26 men and 51 women, with a mean (SD) age of 56 (12) years (range 14-78 years). All patients had recurrent clinical paroxysmal supraventricular tachycardia for a mean period of 4.2 (3.5) years (range 1-10.5 years). Few patients had organic heart disease: two patients had single vessel coronary artery disease, one patient had rheumatic heart disease status post-mitral valve replacement, and two patients had hypertensive heart disease.

For the phase II study, another 51 consecutive patients with proven AVNRT were enrolled prospectively between October 1997 and August 1998. There were 14 men and 37 women, with a mean age of 52 (18) years (range 23-78 years). One patient had two vessel coronary artery disease, one had concurrent right atrial tachycardia, and one had rheumatic heart disease status post-aortic valve replacement.

ELECTROPHYSIOLOGIC STUDY AND SLOW

PATHWAY CATHETER ABLATION

The patients were studied in a fasting, non-sedative state after informed written consent was obtained. All antiarrhythmic drugs were discontinued for at least five half lives. During the study, standard $5 \mathrm{~mm}$ or $2-5-2 \mathrm{~mm}$ spacing quadripolar electrodes (Mansfield EP, Boston Scientific, Watertown, Massachusetts, USA) were positioned at the high right atrium, the His bundle site, and the right ventricular apex. Another decapolar catheter with a central lumen (Daig Corp, Minnetonka, Minnesota, USA) was inserted into the coronary sinus, with the proximal bipole located at the orifice. The coronary sinus catheter was used for both local electrogram recording and direct coronary sinus venography. The His bundle recording catheter was carefully adjusted to record the discrete His potential with a local amplitude ratio of atrial versus ventricular electrogram of about $0.25-0.5$. Every effort was made to maintain the His bundle catheter in a stable position throughout the study.

Standard electrophysiologic study incorporating programmed electrical stimulation up to double extrastimuli at two driving cycle lengths (usually 600 or $500 \mathrm{~ms}$ and $400 \mathrm{~m}$ ) and incremental pacing was performed from both the high right atrium and the right ventricular apex to reproduce the clinical tachycardia. If necessary, isoproterenol infusion would be used to facilitate the induction. Multiple surface ECG leads and intracardiac electrograms were displayed simultaneously and recorded on a computerised oscilloscopic system (Cardiolab, Prucka Engineering Inc, Houston, Texas) with a data sampling rate of $1 \mathrm{kHz}$. Relevant intervals and amplitude measurements were calculated at a speed of 100 or $200 \mathrm{~mm} / \mathrm{s}$ by the calibrated caliper of the system. Pacing stimuli were delivered by a digital stimulator (Bloom Associates, Reading, Pennsylvania, USA) at twice the diastolic threshold in strength and $2 \mathrm{~ms}$ in duration. The diagnosis of AVNRT was established according to the previously suggested criteria. ${ }^{3-5}$

In the phase I study, RF catheter ablation of the slow pathway was performed by dividing the tricuspid border of Koch's triangle into six areas (fig 1), labelled as $\mathrm{A}$ (anterior) ${ }_{1}, \mathrm{~A}_{2}$, $\mathrm{M}$ (middle) ${ }_{1}, \mathrm{M}_{2}, \mathrm{P}$ (posterior) ${ }_{1}$ and $\mathrm{P}_{2}$. The RF catheter ablation site was selected by the stepwise anatomical approach suggested by Jazayeri and colleagues ${ }^{4}$ and started from the site below the floor level of the coronary sinus orifice- that is, the $\mathrm{P}_{2}$ site. The coronary sinus orifice and the tricuspid border were verified by direct coronary sinus venography (fig $2 \mathrm{~A}$ ).

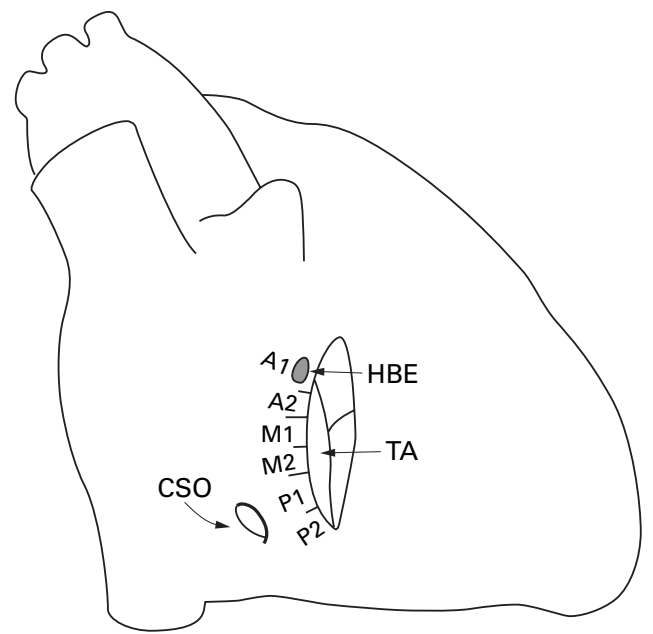

Figure 1 Anatomical scheme for stepwise anatomy guided slow pathway catheter ablation of AVNRT. The tricuspid annular border (TA) between the His bundle recording site (HBE) and the floor level of the coronary sinus orifice (CSO) was arbitrarily separated into anterior $\left(A_{1}, A_{2}\right)$, middle $\left(M_{1}, M_{2}\right)$, and posterior $\left(P_{1}, P_{2}\right)$ regions. The $P_{2}$ region was located below the CSO floor. The slow pathway catheter ablation began at the $P_{2}$ region. If ineffective, the ablation site would be moved anteriorly step by step. The scheme is in right anterior oblique (RAO) view. 
Radiofrequency energy was first applied close to the tricuspid border, with an atrial to ventricular local electrogram amplitude ratio of $0.2-0.5$. If ineffective, another RF pulse would be applied at a slightly more atrial site, with a nearly equal amplitude of atrial and ventricular local electrograms, before moving to the more anterior level. The slow pathway catheter ablation was undertaken in sinus rhythm with continuous monitoring of surface ECG and intracardiac local electrograms. Biplane fluoroscopic imagings were intermittently taken to check the stable positioning of the tip of the ablation catheter during RF energy application. If junctional ectopies or junctional tachycardia occurred, high right atrial overdrive pacing was delivered to monitor the integrity of $\mathrm{AV}$ conduction while application of RF energy continued. The pacing cycle length (usually $450-600 \mathrm{~ms}$ ) was preselected to make sure of stable 1:1 AV conduction. If any AV block developed, the RF energy delivery was immediately interrupted and the electrode at the ablation site would be kept and imaged for record (as described below). Repeated electrophysiologic tests for the inducibility of AVNRT were

A

RAO

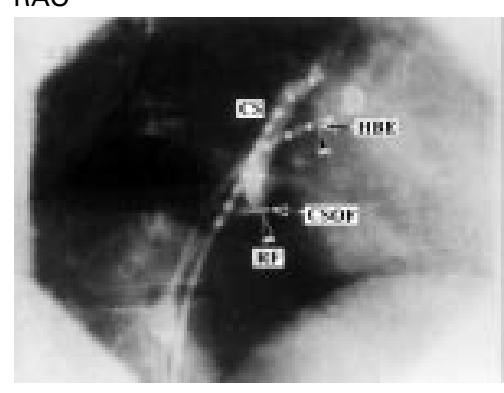

LAO

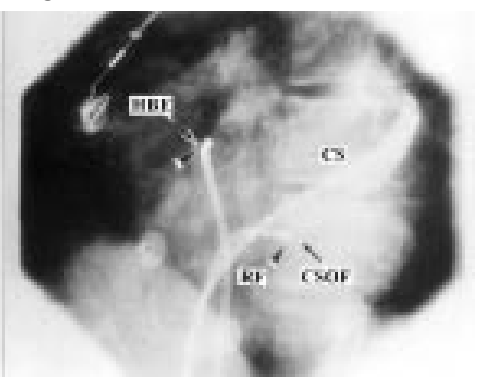

B RAO

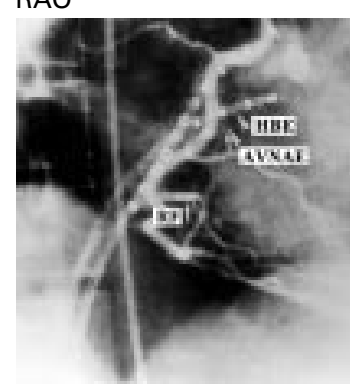
Figure 2 Angiographic study and measurements of anatomical parameters within Koch's
triangle in a patient from group B in whom AVNRT was successfully eliminated by slow pathway catheter ablation during phase I of the study. (A) Biplane direct coronary sinus venography for identification of the coronary sinus (CS) and its orifice, and the proper positioning of the ablation catheter tip before slow pathway catheter ablation. (B) Biplane selective right coronary arteriography (RCA) after successful catheter ablation to demonstrate the $A V$ nodal artery and its ending ( $A V N A E)$ together with the His bundle recording catheter (HBE, discrete His potential recorded by pole 2 and 3 in this patient) and the tip of the ablation catheter (RF). The measurement of the tricuspid border distance between the HBE and the coronary sinus orifice floor (CSOF) levels (black arrow pair), and that between the HBE and the RF catheter ablation site (RF) (black arrowhead pair) are shown in panel $A$; the measurement of the distance between the HBE and the AVNAE (arrowhead pair), and between the AVNAE and the RF catheter ablation site (white arrow pair in RAO, black arrow pair in LAO view), is shown in panel B. The measurement was the straight distance between the tips of each pair of arrows or arrowheads on right anterior oblique (RAO) $45^{\circ}$ or left anterior oblique (LAO) $45^{\circ}$ view. The data were calibrated by the standard spacing of electrode catheters. This patient had successful slow pathway catheter ablation at $P_{2}$ site, which was $15 \mathrm{~mm}$ from the distal end of the AV nodal artery on $R A O$ view and $18 \mathrm{~mm}$ on $L A O$ view. High right atrial and right ventricular apical electrode catheters were removed temporarily to help demonstrate the anatomical landmarks. conducted after each application of RF energy. Each application of RF energy was preset for a total of 60 seconds with the catheter tip temperature targeted at $60^{\circ} \mathrm{C}$. Radiofrequency current $(500 \mathrm{kHz})$ was delivered between the $4 \mathrm{~mm}$ distal electrode of the ablation catheter imbedded with a tip thermistor (Dr Osypka GmbH, Grenzach-Wyhlen, Germany or Webster-Cordis, Orlando, Florida, USA) and a large dispersive patch electrode on the back. The RF generator (HAT 200S, Dr Osypka $\mathrm{GmbH}$, Berlin, Germany) was commercially available with the power, voltage, current, impedance, and temperature monitorings connected to the computerised oscilloscopic recorder. The end point of the slow pathway catheter ablation was the non-inducibility of all forms of AVNRTs and elimination or modification of the AV nodal slow pathway, which was defined as the persistence of the dual AV nodal physiology and the induction of a single AV nodal echo beat.

In the phase II study, the slow pathway catheter ablation was guided by localisation of the His bundle recording site, the coronary sinus orifice, and the distal end of the AV nodal artery demonstrated by coronary angiography before the ablation procedure. Every effort was made to keep the ablation target site posterior to and away from the distal end of the AV nodal artery. Radiofrequency energy was applied close to the tricuspid border, with smaller atrial local electrogram at the target site. At the end of the session, the final ablation site was recorded with a repeat selective coronary angiography to confirm further the anatomic spatial relation.

\section{ANGIOGRAPHIC STUDY}

In phase I, the final target sites of successful slow pathway catheter ablation or the sites complicated with transient or permanent complete AV block were imaged simultaneously with selective right or left coronary angiography (fig 2B). However, in phase II, selective coronary angiography for demonstration of the AV nodal artery was performed before the selection of target sites for slow pathway catheter ablation. All the angiographic images were taken in biplane left $45^{\circ}$ and right $45^{\circ}$ anterior oblique (LAO, RAO) views with the same magnification (9 inches $(22.5 \mathrm{~cm})$ ) during the end expiratory breath holding period. The spatial resolution of the angiographic imaging is $0.5 \mathrm{~mm}$.

For analysing the anatomical relation, the His bundle recording bipole was referred to as the apex of the Koch's triangle at the AV junction, while the floor level of the coronary orifice was the base of the triangle. The AV nodal artery was identified as a long septal branch arising from the $U$ loop of the posterolateral branch of the right coronary artery or from the left circumflex artery at the crux of the heart. The critical distal end of the AV nodal artery was defined as the identifiable straight ending of the AV nodal artery under 9 inch magnification films. In the event of fork shaped or double stranded arterial endings, the distal end of the AV nodal artery was taken at the midpoint 
between the two ends of the artery. Of the 128 patients with AVNRT in the phase I and phase II studies, $113(88.3 \%)$ had a single AV nodal artery ending, $13(10.1 \%)$ had a fork shaped ending, and two $(1.6 \%)$ had a double stranded ending. The AV nodal artery originated from the right coronary artery in $118(92.2 \%)$ patients and from the left circumflex artery in $10(7.8 \%)$. None had double or more AV nodal arteries from the same or different coronary arteries. All of the selective coronary angiography procedures were completed smoothly without any complication.

ELECTROPHYSIOLOGIC AND ANATOMICAL

PARAMETERS PREDICTING THE RISK OF AV BLOCK To examine the potential predictors for the risk of AV block during slow pathway catheter ablation in the phase I study, multiple electrophysiologic and anatomical parameters were independently analysed by authors from the two institutions without knowing the results of the procedures (JL Lin and LP Lai versus LJ Lin and $\mathrm{JH}$ Chen). If there was any controversy in the data measurement, consensus would be obtained from all four analysers to achieve resolution.

Electrophysiologic parameters included the retrograde atrial activation time difference between the His bundle recording site and the coronary sinus orifice in AVNRT $\left(\mathrm{A}_{\mathrm{HB}}{ }_{\mathrm{HB}}-\mathrm{A}_{\mathrm{CSO}}{ }\right){ }^{4}$ the antegrade atrial activation time difference between the His bundle recording site and the final catheter ablation site in sinus rhythm $\left(\mathrm{A}_{\mathrm{HB}}-\mathrm{A}_{\mathrm{MAP}}\right),{ }^{12}$ and the elapsed time before the onset of junctional tachycardia during RF application. The mean cycle length of the junctional tachycardia and the occurrence of retrograde VA block with junctional ectopies were not systematically analysed because of the routine use of atrial overdrive pacing to monitor the AV conduction.

Anatomical parameters included the following spatial distances: (1) between the distal end of the AV nodal artery and the final catheter ablation site; (2) between the His bundle recording site and the tricuspid border at the floor level of the coronary sinus orifice (that is, the tricuspid border dimension of Koch's triangle); (3) between the His bundle recording site and the final catheter ablation site; and (4)

Table 1 Comparison of clinical, electrophysiologic, and ablation characteristics in nine patients with (group A) and 68 patients without (group B) AV block in the phase I study

\begin{tabular}{llll}
\hline & $\begin{array}{l}\text { Group } A \\
n=9\end{array}$ & $\begin{array}{l}\text { Group } B \\
n=68\end{array}$ & p Value \\
\hline Mean (SD) age (years) & $59(15)$ & $55(16)$ & 0.53 \\
Sex (male/female) & $2 / 7$ & $24 / 44$ & 1.69 \\
Mean (SD) AVNRT cycle length (ms) & $355(66)$ & $373(41)$ & 0.25 \\
AVNRT forms & & & 0.40 \\
S-F & 9 & 62 & \\
F-S & 0 & 4 & \\
S-F and F-S & 0 & 2 & 0.10 \\
RFCA site & 2 & 3 & \\
$M_{1}$ & 3 & 12 & \\
$M_{2}$ & 3 & 43 & \\
$P_{1}$ & 1 & 10 & 0.67 \\
$P_{2}$ & $3.5(1.8)$ & $3.2(2.0)$ & 0.70 \\
Mean (SD) number of RFCA applications & $2.2(1.0)$ & $2.0(1.5)$ & \\
RFCA time (hours) & & & \\
\hline
\end{tabular}

AVNRT, atrioventricular nodal re-entrant tachycardia; S-F, F-S, slow-fast, fast-slow form of AVNRT, in patient number; RFCA, radiofrequency catheter ablation; $M_{1}, M_{2}, P_{1}, P_{2}$, midseptal and posteroseptal sites of Koch's triangle (see fig 1); RFCA time, total procedure duration. between the His bundle recording site and the distal end of the AV nodal artery (fig 2A, B). All the anatomical parameters were measured immediately before the rapid ventricular filling phase or in the late systolic phase validated by concomitant ECG monitoring, while atrial volume was expected to be full. The regular spacing of the mapping electrodes was used as a standard for calibration and measurement of the anatomical parameters.

\section{STATISTICS}

Continuous data were compared by unpaired Student's $t$ test and categoric data by $\chi^{2}$ test with Yates' correction. Stepwise univariate and multivariate logistic regression analysis was employed to analyse the independent predictor(s) of risk of AV block during slow pathway catheter ablation. A value of $p<0.05$ was considered significant.

\section{Results}

SLOW PATHWAY CATHETER ABLATION OF AVNRT IN THE PHASE I STUDY

In the phase I study, AVNRT was successfully ablated in $75(97.4 \%)$ of the 77 patients by either slow pathway complete elimination (60 patients) or modification (15 patients). In two patients $(2.6 \%)$, RF catheter ablation was complicated with irreversible complete AV block and a permanent pacemaker was implanted. In addition, in seven of the 75 patients with a successful outcome, the procedure was associated with transient high grade AV block requiring immediate interruption of RF energy application. The duration of transient AV block before full recovery of the normal AV conduction in the seven patients lasted from 5-120 seconds (mean 53.3 (38.3) seconds).

Nine patients complicated with reversible or irreversible AV block were classified as group A, while the remaining 68 patients without the complication were classified as group $\mathrm{B}$. The time which elapsed before the appearance of transient or permanent complete AV block during RF energy delivery in the nine group A patients ranged from 12-90 seconds (mean 37.0 (27.9) seconds). One patient developed a period of transient complete AV block 30 seconds after completion of the preset ablation time.

The site of complete AV block was supraHisian in all patients. Clinical and electrophysiologic characteristics including age, sex, details of induced AVNRT, distribution of the final RF catheter ablation sites within Koch's triangle, total number of RF applications, and total procedure time were similar between groups $\mathrm{A}$ and B (table 1).

ELECTROPHYSIOLOGIC PREDICTORS OF THE RISK OF AV BLOCK IN THE PHASE I STUDY

As shown in table 2, none of the available electrophysiologic parameters either before or during RF energy application were significant in predicting the risk of $\mathrm{AV}$ block during slow pathway catheter ablation in the phase I study. An example of the local electrograms at the ablation target sites in a group A patient is shown in fig 3 . However, the atrial activation 
Table 2 Comparison of warning electrophysiologic predictors noted during slow pathway catheter ablation in nine patients with (group $A$ ) and 68 patients without (group B) $A V$ block in the phase I study

\begin{tabular}{|c|c|c|c|c|c|}
\hline & $A_{H B}^{\prime}-A_{C S O}^{\prime}$ & $A_{H B}-A_{M A P}$ & $A / V$ & $\mathscr{f} T / \mathscr{f} E$ & $\mathcal{F T}$ onset \\
\hline Group A & $9.6 \quad(8.3)$ & $14.2(4.6)$ & $0.4(0.6)$ & $9 / 9$ & $9.4 \quad(12.8)$ \\
\hline Group B & $13.0(8.2)$ & $19.7 \quad(9.2)$ & $0.7(0.6)$ & $59 / 68$ & 10.0 \\
\hline p Value & 0.25 & 0.08 & 0.16 & 0.73 & 0.29 \\
\hline
\end{tabular}

$\mathrm{A}_{\mathrm{HB}}^{\prime}-\mathrm{A}_{\mathrm{CSO}}{ }_{\mathrm{CO}}$, retrograde atrial activation time difference (in $\mathrm{ms}$ ) between His bundle recording site (HB) and coronary sinus orifice (CSO) during AVNRT; $\mathrm{A}_{\mathrm{HB}}-\mathrm{A}_{\mathrm{MAP}}$, atrial activation time interval (in ms) between $\mathrm{HB}$ and RF catheter ablation site (MAP) in sinus rhythm; $\mathrm{A} / \mathrm{V}$, atrial versus local ventricular electrogram amplitude ratio; JT/JE, junctional ectopy and tachycardia, in patient number; JT onset, onset time of JT (in seconds) after the delivery of RF energy. The numbers in the parenthesis represent the standard deviation of the mean value.

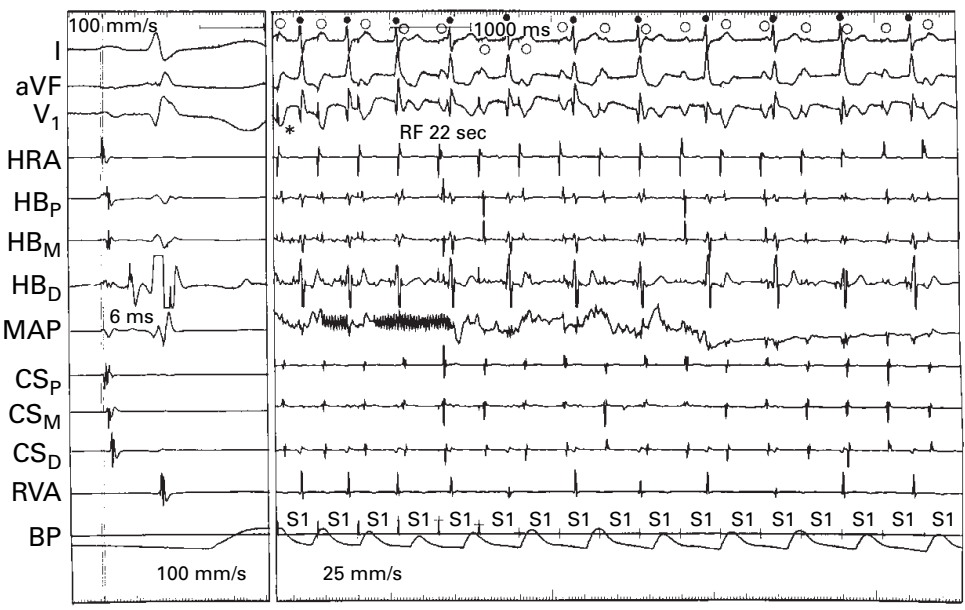

Figure 3 Simultaneous surface ECG and intracardiac electrograms immediately before (left panel) and during application of $R F$ energy (right panel) in a group $A$ patient complicated with permanent complete AV block in phase I of the study during slow pathway catheter ablation of AVNRT. The antegrade atrial activation time difference between the $H B_{D}$ and the catheter ablation target site (MAP) in sinus rhythm was 6 ms. No definite His potential was observed at the selected target site ( $P$ location). Complete AV block developed suddenly after the delivery of $R F$ energy for 22 seconds ( $R F 22$ secs). The $R F$ energy was immediately shut off. However, the AV conduction never recovered. In the right panel, solid dots representing QRS complexes and empty dots representing $P$ waves were marked to show the rapid progression of $A V$ block after a normally conducting paced beat (asterisk). BP, blood pressure; $C S_{P}, C S_{M}, C S_{D}$, proximal (9-10 pair), middle (5-6 pair), and distal (1-2 pair) bipolar electrograms from a decapolar coronary sinus (CS) catheter $H B_{P}, H B_{M}, H B_{D}$, proximal (3-4 pair), middle (2-3 pair), and distal (1-2 pair) bipolar electrograms from the His bundle $(H)$ recording quadripolar catheter (5 $\mathrm{mm}$ spacing); $R V A$, right ventricular apex. time difference between the His bundle recording site and the $\mathrm{RF}$ catheter ablation site in sinus rhythm was shorter in group A patients (14.2 (4.6) $\mathrm{ms}$ in group A $v 19.7$ (9.2) $\mathrm{ms}$ in group $\mathrm{B}, \mathrm{p}=0.08)$.

ANATOMICAL PREDICTORS OF THE RISK OF AV BLOCK IN THE PHASE I STUDY

The fluoroscopic locations of the catheter ablation sites in the nine patients in group A complicated with AV block were found in the $\mathrm{P}_{2}$ region in one patient, $\mathrm{P}_{1}$ region in three, $\mathrm{M}_{2}$ region in three, and $M_{1}$ region in two. In the 68 patients in group $\mathrm{B}$ with successful ablation, 10 sites were in the $\mathrm{P}_{2}$ region, 43 in the $\mathrm{P}_{1}$ region, 12 in the $M_{2}$ region and three in the $M_{1}$ region $\left(\mathrm{p}=0.10\right.$, by $\chi^{2}$, table 1$)$. However, as shown in table 3, compared to those in group $\mathrm{B}$, the anatomical parameters in group A patients characteristically had a wider distance between the His bundle recording site and the distal end of the AV nodal artery (group A $v$ group B in RAO view: $11.3(6.8) \mathrm{mm} v 6.8$ (3.7) $\mathrm{mm}$, $\mathrm{p} \leqslant 0.003$; in LAO view: 11.0 (4.7) $\mathrm{mm} v 7.7$ (3.9) $\mathrm{mm}, \mathrm{p} \leqslant 0.02$ ) and a nearly overlapped approximation of the final catheter ablation site and the distal end of the AV nodal artery (group A $v$ group B in RAO view: 0.61 $(0.60) \mathrm{mm} v 7.2(4.0) \mathrm{mm}, \mathrm{p} \leqslant 0.00005$; in LAO view: $0.56(0.58) \mathrm{mm} v 6.9(4.0) \mathrm{mm}$, $\mathrm{p} \leqslant 0.00007)$. Examples of the anatomical landmarks for two patients in group $\mathrm{A}$ and one patient in group B are shown in figs 4 and 5, and fig $2 \mathrm{~B}$. Sequential univariate and multivariate regression analysis revealed that only the anatomical distance between the catheter ablation site and the distal end of the AV nodal artery was independently predictive of the risk of AV block during slow pathway catheter ablation of AVNRT. All of the nine patients in group A, but none of the 68 patients in group $\mathrm{B}$, had a closely approximated distance of $<2 \mathrm{~mm}$ between the catheter ablation site and the AV nodal artery ending.

The success site without AV block was 7.2 (4.0) $\mathrm{mm}$ (RAO view) and 6.9 (4.0) $\mathrm{mm}$ (LAO view) away from the distal end of the AV nodal artery, and 14.1 (4.3) $\mathrm{mm}$ (RAO view) and 14.4 (3.5) $\mathrm{mm}$ (LAO view) from the His bundle recording site (table 3 ).

demonstrated by angiography in nine patients with (group A) and 68 patients without (group B) AV block during slow pathway catheter ablation of AVNRT in the phase I study

\begin{tabular}{|c|c|c|c|c|}
\hline & Group $A$ & Group B & $p_{1}$ & $p_{2}$ \\
\hline \multicolumn{5}{|l|}{ RAO $45^{\circ}$ view } \\
\hline $\mathrm{D}_{\mathrm{HB}-\mathrm{CSOF}}$ & $18.8 \quad(4.2)$ & $20.1(5.3)$ & 0.51 & - \\
\hline$D_{\text {HB-AVNAE }}$ & $11.3(6.8)$ & $6.8(3.7)$ & 0.003 & 0.11 \\
\hline $\mathrm{D}_{\mathrm{HB}-\mathrm{RF}}$ & $11.7 \quad(6.8)$ & $14.1(4.3)$ & 0.14 & - \\
\hline $\mathrm{D}_{\mathrm{AVNAE}-\mathrm{RF}}$ & $0.61(0.60)$ & $7.2(4.0)$ & 0.00005 & 0.001 \\
\hline \multicolumn{5}{|l|}{ LAO $45^{\circ}$ view } \\
\hline $\mathrm{D}_{\mathrm{HB}-\mathrm{CSOF}}$ & $20.6 \quad(5.9)$ & $23.6(4.6)$ & 0.08 & - \\
\hline $\mathrm{D}_{\mathrm{HB}-\mathrm{AVNAE}}$ & $11.0 \quad(4.7)$ & $7.7(3.9)$ & 0.02 & 0.43 \\
\hline $\mathrm{D}_{\mathrm{HB}-\mathrm{RF}}$ & $12.8 \quad(5.2)$ & $14.4(3.5)$ & 0.23 & - \\
\hline $\mathrm{D}_{\text {AVNAE-RF }}$ & $0.56(0.58)$ & $6.9(4.0)$ & 0.00007 & 0.001 \\
\hline
\end{tabular}

AVNAE, distal end of AV nodal artery; CSO, coronary sinus orifice; CSOF, CSO floor; D, distance or dimension (in $\mathrm{mm}$ ); $\mathrm{D}_{\mathrm{HB}-\mathrm{CSOF}}, \mathrm{D}_{\mathrm{HB}-\mathrm{AVNAF}}, \mathrm{D}_{\mathrm{HB}-\mathrm{RF}}$, distance between His bundle recording site (HB) and CSOF, AVNAE, and RF catheter ablation site (RF); $\mathrm{D}_{\text {AVNAE-RF }}$, distance between AVNAE and RF; $\mathrm{p}_{1}$, $\mathrm{p}$ value between group A and group B, by Student's $t$ test; $\mathrm{p}_{2}, \mathrm{p}$ value by multivariate regression analysis; $p<0.05$ is significant. The number in the parenthesis represents the standard deviation of the mean value.
SUCCESS, COMPLICATION, AND ANATOMICAL LOCATIONS OF SLOW PATHWAY CATHETER ABLATION OF AVNRT IN THE PHASE II STUDY Of 51 patients with AVNRT in the phase II study, the slow-fast type was found in 47 patients, fast-slow type in two, and both slow-fast and fast-slow type in two. The mean cycle length of the induced AVNRT was 360 (42) ms (range 242-510 ms). Slow pathway catheter ablation guided by the three anatomical landmarks in Koch's triangle- that is, the His bundle recording site, the coronary sinus orifice, and the distal end of the AV nodal artery- successfully eliminated all forms of AVNRT without AV block. The slow pathway conduction of the AV node was eliminated in 35 patients and modified in 16 . None of the 51 patients had inadvertent fast pathway damage or modification. 
RAO

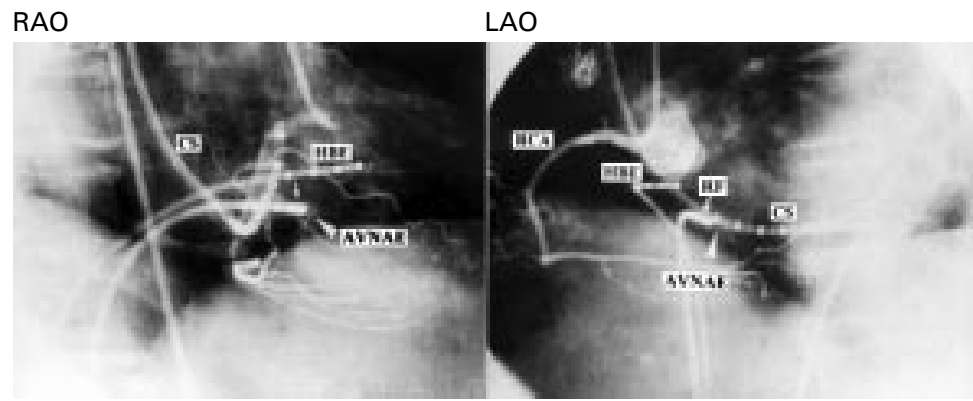

Figure 4 Fluoroscopic illustration showing the close approximation of the distal end of the $A V$ nodal artery and the tip of the ablation catheter at $M_{2}$ region in a group $A$ patient from the phase I study, complicated with transient complete AV block during slow pathway catheter ablation. Immediate interruption of $R F$ energy delivery restored the normal $A V$ conduction in 45 seconds. The spatial distance between the AV nodal artery ending ( $A V N A E$, arrowheads) and the tip of the ablation catheter (RF, small arrows) is $<1 \mathrm{~mm}$. See fig 2 caption for other abbreviations.

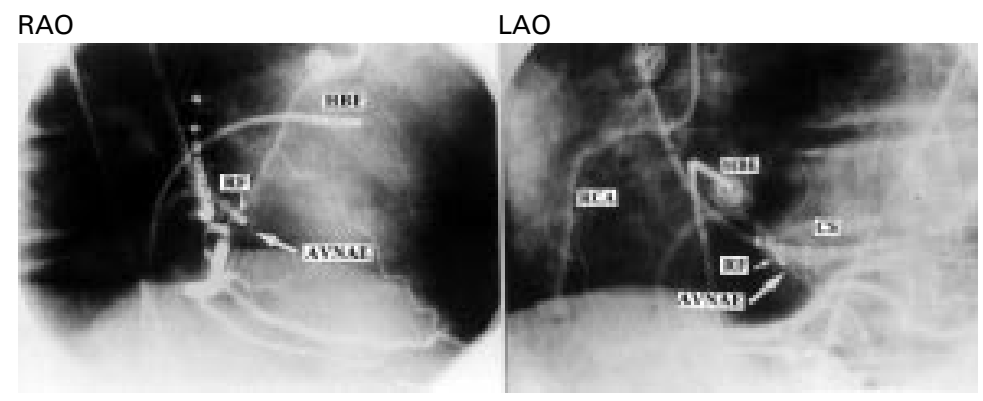

Figure 5 Fluoroscopic illustration showing the spatial relation between the distal end of the $A V$ nodal artery and the tip of the ablation catheter at $P_{1}$ region in a group $A$ patient from the phase I study complicated with permanent complete AV block. The distance between the $A V$ nodal artery ending ( $A V N A E$, large arrows) and the tip of the ablation catheter (RF, small arrows) is $<1 \mathrm{~mm}$. Note the very short AV nodal artery originating from the right coronary artery. See fig 2 caption for other abbreviations.

The fluoroscopic locations of the successful ablation sites were found in the $\mathrm{P}_{2}$ region in seven patients, $P_{1}$ region in $28, M_{2}$ region in 14 , and $M_{1}$ region in two. The distal ends of the AV nodal artery were located in the $\mathrm{A}_{1}$ region in $13, A_{2}$ region in $10, M_{1}$ region in 13 , $M_{2}$ region in $10, P_{1}$ region in four, and $P_{2}$ region in one. Final angiographic reconfirmation showed that the spatial distance between the catheter ablation site and the distal end of the AV nodal artery was $8.8(4.2) \mathrm{mm}(v 7.2$ (4.0) $\mathrm{mm}$ for group B in phase $\mathrm{I}, \mathrm{p}=0.03)$ in RAO view and $9.2(5.1) \mathrm{mm}(v 6.9(4.0) \mathrm{mm}$ in phase $\mathrm{I}, \mathrm{p}=0.007$ ) in LAO view, while that between the ablation site and the His

Table 4 Comparison of anatomical parameters demonstrated by angiography in 68 phase I and 51 phase II patients with successful and uncomplicated slow pathway catheter ablation of $A V N R T$

\begin{tabular}{|c|c|c|c|}
\hline & $\begin{array}{l}\text { Phase } \\
\text { I } n=68\end{array}$ & Phase II $n=51$ & $p$ Value \\
\hline \multicolumn{4}{|l|}{ RAO $45^{\circ}$ view } \\
\hline $\mathrm{D}_{\mathrm{HB}-\mathrm{CSOF}}$ & $20.1(5.3)$ & $21.2(6.0)$ & 0.29 \\
\hline $\mathrm{D}_{\mathrm{HB}-\mathrm{AVNAE}}$ & $6.8(3.7)$ & $6.4(2.8)$ & 0.53 \\
\hline $\mathrm{D}_{\mathrm{HB}-\mathrm{RF}}$ & $14.1(4.3)$ & $15.8(4.7)$ & 0.04 \\
\hline $\mathrm{D}_{\text {AVNAE-RF }}$ & $7.2(4.0)$ & $8.8(4.2)$ & 0.03 \\
\hline \multicolumn{4}{|l|}{ LAO $45^{\circ}$ view } \\
\hline $\mathrm{D}_{\mathrm{HB}-\mathrm{CSOF}}$ & $23.6(4.6)$ & $22.6(5.6)$ & 0.29 \\
\hline $\mathrm{D}_{\mathrm{HB}-\mathrm{AVNAE}}$ & $7.7(3.9)$ & $6.8(2.9)$ & 0.17 \\
\hline $\mathrm{D}_{\mathrm{HB}-\mathrm{RF}}$ & $14.4(3.5)$ & $16.0(3.8)$ & 0.02 \\
\hline $\mathrm{D}_{\mathrm{AVNAE}-\mathrm{RF}}$ & $6.9(4.0)$ & $9.2(5.1)$ & 0.007 \\
\hline
\end{tabular}

$\mathrm{D}$, distance or dimension (in $\mathrm{mm}$ ); $\mathrm{D}_{\mathrm{HB}-\mathrm{CSOF}}, \mathrm{D}_{\mathrm{HB}-\mathrm{AVNAE}}, \mathrm{D}_{\mathrm{HB}-\mathrm{RF}}$, $\mathrm{D}_{\text {AVNAE-RF}}$, defined in table $3 ; \mathrm{p}, \mathrm{p}$ value between phase I and phase II studies, by Student's $t$ test; $\mathrm{p}<0.05$ is significant. The numbers in parenthesis represent the standard deviation of the mean value. bundle recording site was $15.8(4.7) \mathrm{mm}$ ( $v$ 14.1 (4.3) $\mathrm{mm}$ for group $\mathrm{B}$ in phase $\mathrm{I}$, $\mathrm{p}=0.04)$ in RAO view and $16.0(3.8) \mathrm{mm}(v$ 14.4 (3.5) $\mathrm{mm}$ in phase $\mathrm{I}, \mathrm{p}=0.02)$ in LAO view. The distances from the His bundle recording site to the distal end of the AV nodal artery and to the floor level of the coronary sinus orifice were similar to those in the phase I study (table 4).

\section{Discussion}

MAIN FINDINGS

This study showed that the anatomical approximation of the ablation target site to the distal end of the AV nodal artery is highly and independently predictive of the risk of AV block during slow pathway catheter ablation for the treatment of AVNRT. A spatial distance $<2 \mathrm{~mm}$ between the ablation target site and the distal end of the AV nodal artery almost always resulted in supra-Hisian AV block. Thus, the distal end of the AV nodal artery apparently serves as a useful landmark for angiographic localisation of the AV node within Koch's triangle. In addition, patients complicated with transient or permanent complete AV block characteristically had a more posteriorly located AV node. In contrast, none of the electrophysiologic signals either before or during RF energy application were predictive of the occurrence of AV block.

AV NODAL ARTERY AND AV NODE

The AV nodal artery which supplies the AV node $^{14}{ }^{15}$ usually originates as a septal branch from the distal right coronary artery proper (or uncommonly from the left circumflex artery proper) at the crux of the heart in humans. The artery penetrates toward the interatrial septum and tapers to $<0.4 \mathrm{~mm}$ in internal diameter before entering the compact AV node. ${ }^{15-17}$ Inside the compact node, the AV nodal artery directly transforms into minute plexiform arterioles and communicates with the vascular network around the His bundle. Previous experimental ${ }^{16}$ and clinical $^{17}{ }^{18}$ studies have shown that selective catheterisation of the AV nodal artery and direct infusion of contrast medium, acetylcholine, alcohol concentrate or collagen emboli consistently results in complete AV block in the node, not at or below the His bundle. The selectivity of the anatomical site of injury leads to our hypothesis that the distal end of the AV nodal artery could serve as the anatomical landmark for localisation of the AV node within Koch's triangle.

ELECTROPHYSIOLOGIC PARAMETERS VERSUS ANATOMICAL LANDMARKS FOR PREDICTING THE RISK OF AV BLOCK

Current practice for prevention of inadvertent AV block relies primarily on the analysis and continuous monitoring of local electrophysiologic parameters either before or during slow pathway catheter ablation. Jazayeri and colleagues $^{4}$ first found that the retrograde atrial activation time difference between the His bundle site and the coronary sinus orifice $\left(\mathrm{A}_{\mathrm{HB}}^{\prime}-\mathrm{A}_{\mathrm{CSO}}^{\prime} \leqslant 15 \mathrm{~ms}\right)$ during AVNRT or 
during ventricular pacing predicted a high risk of complete $\mathrm{AV}$ block during fast pathway catheter ablation, and suggested a posteriorly located fast pathway in Koch's triangle. Engelstein and colleagues ${ }^{19}$ recently reported similar risk of complete AV block during slow pathway catheter ablation targeted at the posteroseptal atrial septum for a specific group of patients who have a reverse or simultaneous retrograde atrial activation pattern at the His bundle site and the coronary sinus orifice. However, the retrograde fast pathway conduction pattern in patients with typical AVNRT is probably never discrete, and is subject to variations in the activation sequence during tachycardia versus during ventricular pacing because of the anatomical heterogeneity of the functional anterior atrionodal inputs. ${ }^{20}{ }^{21}$ Hintringer and colleagues ${ }^{12}$ thus proposed that a short atrial activation time difference in sinus rhythm between the His bundle region and the distal mapping electrodes $\left(\mathrm{A}_{\mathrm{HB}}-\mathrm{A}_{\mathrm{MAP}} \leqslant 20 \mathrm{~ms}\right)$ is a better warning sign before RF energy delivery for slow pathway catheter ablation. On the other hand, during delivery of RF energy, Thakur and colleagues $^{13}$ felt that the occurrence of fast junctional tachycardia (mean cycle length 363 (44) $\mathrm{ms}$ ) with retrograde VA block is a sign of impending $\mathrm{AV}$ block during slow and fast pathway catheter ablation of AVNRT. However, the reliability of junctional tachycardia as a risk indicator is largely depreciated by other studies $^{912}$ which show a low specificity (for example, junctional tachycardia rate) and a low positive predictive value (for example, junctional ectopies with VA block).

Our study did not support the predictive efficacy of the aforementioned electrophysiologic parameters. Instead, with the help of biplane angiographic studies, we found that identification of anatomical landmarks for predicting the risk of AV block is feasible. The distal end of the AV nodal artery serves well as a useful landmark for anatomical localisation of the AV node and accurately predicts the risk of AV block during slow pathway catheter ablation. In addition, although not as critical, the $\mathrm{AV}$ node location, represented by the AV nodal artery ending, is more posteriorly displaced in patients complicated with transient or permanent complete AV block. In our opinion, angiographic identification of these anatomical landmarks, especially the spatial relation between the distal end of the AV nodal artery and the ablation site, is a reliable approach for predicting the risk of AV block during slow pathway catheter ablation of AVNRT.

LIMITATIONS

First, the clinical applicability of this anatomical landmark - that is, the distal end of the AV nodal artery-in the slow pathway RF catheter ablation of AVNRT could be limited by the increased cost and potential risk associated with the additional coronary angiography. ${ }^{22}$ However, our study at least showed the feasibility and reliability of using such a new landmark in identification of the site at high risk of AV block during RF ablation of AVNRT by the posterior approach. It might be helpful in patients with an unusual functional anatomy of Koch's triangle - for example, the posteriorly deviated AV node or fast pathway. ${ }^{19}$ Second, because of the use of atrial overdrive pacing to monitor the integrity of AV conduction in the present study, the usefulness of junctional ectopies with retrograde VA block ${ }^{9}{ }^{12}$ during RF application cannot be evaluated and cannot be compared with that of our proposed anatomical landmark. Furthermore, it might be too late to avoid AV block at the time when VA block occurs during junctional tachycardia. Third, the subtle details of the distal end of the AV nodal artery-for example, the plexiform connections with the peri-His bundle vascular networks-could not be demonstrated on standard angiography and fluoroscopic magnification. Nevertheless, our phase II study supported the finding that the distal end of the AV nodal artery shown by the standard technique would be sufficient enough to serve as a useful landmark to avoid the risk of AV block. Fourth, only the angiographic locations of the successful ablation sites and the sites complicated with AV block were recorded for analysis. The cumulative effect from previous $\mathrm{RF}$ applications at the failed ablation sites could not be verified.

\section{CONCLUSIONS}

The identification of the distal end of the AV nodal artery by selective coronary angiography is useful for the prediction of AV block during slow pathway catheter ablation of AVNRT. Prior angiographic demonstration of this anatomic landmark before application of RF energy may be an important step for assisting the selection of an appropriate target site for ablation in order to minimise the risk of $\mathrm{AV}$ block.

This study is supported in part by grants from the National Science Council (NSC 87-2314-B-002-193) and the National Health Research Institute (DOH 87-HR-711) in Taiwan. The authors appreciate the technical support from Mr Ming-Tuan Liu and Mr Shin-Jee Ho in angiographic studies and the secretarial assistance of Ms Yueh-Ju Liao and Ms Jen-Yu Huang in preparation of the manuscript. This paper was presented in part at the $47 \mathrm{th}$ annual scientific session of the American College of Cardiology in Atlanta, March, 1998.

1 McGuire MA, Janse MJ, Ross DL. "AV nodal" reentry: Part II: AV nodal, AV junctional, or atrionodal reentry? $\mathcal{f}$ Cardiovasc Electrophysiol 1993;4:573-86.

2 Stein KM, Lerman B. Evidence for functionally distinct dual atrial inputs to the human AV node. Am 7 Physiol 1994;267:H2333-41.

3 Jackman WM, Beckman KJ, McClelland JH, et al. Treatment of supraventricular tachycardia due to atrioventricular nodal reentry by radiofrequency catheter ablation of slow-pathway reentry by radiofrequency catheter ablation
conduction. $N$ Engl F Med 1992;327:313-8.

4 Jazayeri M, Hempe SL, Sra JS, et al. Selective transcatheter ablation of the fast and slow pathways using radiofrequency energy in patients with atrioventricular nodal re-entrant tachycardia. Circulation 1992;85:1318-28.

5 Prystowsky EN. Atrioventricular node reentry: physiology and radiofrequency ablation. PACE 1997;20(part II):55271 .

6 Scheinman MM. NASPE survey on catheter ablation. PACE 1995;18:1474-8

7 Wathen M, Natale A, Wolfe K, et al. An anatomically-guided approach to atrioventricular node slow pathway ablation. Am $\mathcal{F}$ Cardiol 1992;70:886-9.

8 Wu D, Yeh SJ, Wang CC, et al. A simple technique for selective radiofrequency ablation of the slow pathway in atrioventricular node re-entrant tachycardia. $\mathcal{F} \mathrm{Am}$ Coll Cardiol 1993;21:1612-21.

9 Jentzer JH, Goyal R, Williamson BD, et al. Analysis of junctional ectopy during radiofrequency ablation of the slow pathway in patients with atrioventricular nodal re-entrant tachycardia. Circulation 1994;90:2820-6. 
10 Fenelon G, d'Avila A, Malacky T, et al. Prognostic significance of transient complete atrioventricular block during radiofrequency ablation of atrioventricular node re-entrant tachycardia. Am 7 Cardiol 1995;75:698-702.

11 Chen SA, Chiang CE, Tai CT, et al. Transient complete atrioventricular block during radiofrequency ablation of slow pathway for atrioventricular nodal re-entrant tachycardia. Am f Cardiol 1996;77:1367-70.

12 Hintringer F, Hartikainen J, Davies DW, et al. Prediction of atrioventricular block during radiofrequency ablation of the slow pathway of the atrioventricular node. Circulation 1995;92:3490-6.

13 Thakur RK, Klein GJ, Yee R, et al. Junctional tachycardia: a useful marker during radiofrequency ablation for atrioventricular node re-entrant tachycardia. $7 \mathrm{Am}$ Coll Cardiol 1993;22:1706-10.

14 Lumb G, Singletary MD. Blood supply to the atrioventricular node and bundle of His: a comparative study in pig, dog and man. Circulation 1961;24:986-92.

15 Van der Hauwaert LG, Stroobandt R, Verhaeghe L. Arterial blood supply of the atrioventricular node and main bundle. Br Heart $\mathcal{f} 1972 ; 34: 1045-51$.

16 Wang PJ, Shoen FJ, Reagan K, et al. Modification of atrioventricular conduction by selective AV nodal artery catheterization. PACE 1990;13:88-102.
17 Wang PJ, Sosa-Suarez G, Friedman PL. Modification of human atrioventricular nodal function by selective atriov817-29.

18 Kay GN, Bubien RS, Dailey SM, et al. A prospective evaluation of intracoronary ethanol ablation of the atrioventricular conduction system. F Am Coll Cardiol 1991;17:1634-40.

19 Engelstein ED, Stein KM, Markowitz SM, et al. Posterior fast atrioventricular node pathways: implications for radiofrequency catheter ablation of atrioventricular node re-entrant tachycardia. f Am Coll Cardiol 1996;27:1098105.

20 Janse MJ, Anderson RH, McGuire MA, et al. "AV nodal" reentry: part I: "AV nodal" reentry revisited. 7 Cardiovasc Electrophysiol 1993;4:561-72.

21 Anselme F, Hook B, Monahan K, et al. Heterogeneity of retrograde fast-pathway conduction pattern in patients with atrioventricular nodal reentry tachycardia: observawith atrioventricular nodal reentry tachycardia: observa-
tions by use of simultaneous multisite catheter mapping of tions by use of simultaneous multisite cathet
Koch's triangle. Circulation 1996;93:960-8.

22 Scanlon PJ, Faxon DP, Audet A-M, et al. ACC/AHA guidelines for coronary angiography: a report of the American College of Cardiology/American Heart Association task force on practice guidelines (committee on coronary angiography). $\mathcal{F}$ Am Coll Cardiol 1999;33:1756-816.

\section{Strangulated gastric volvulus: an unusual cause of cardiac compression resulting in electromechanical dissociation}

A 50 year old man was admitted with severe epigastric pain. He had previously had an open Heller's cardiomyotomy for achalasia. Subsequent investigations for persisting dyspepsia had demonstrated a rolling hiatus hernia. $\mathrm{He}$ was tachycardic and hypotensive, with a raised jugular venous pressure. His chest radiograph showed bowel loops within the left hemithorax and a left pleural reaction.

An urgent transthoracic echocardiogram revealed that the atria were obscured by an extracardiac mass. A diagnosis of extrinsic cardiac

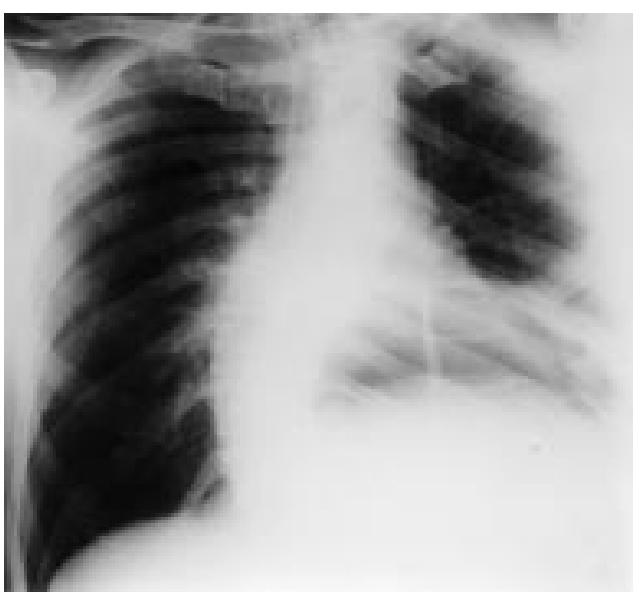

compression secondary to a gastric volvulus was made. While awaiting emergency surgery his condition deteriorated culminating in electromechanical dissociation (EMD). Following successful resuscitation there was an immediate improvement in his blood pressure. At surgery he had a tight, scarred hiatus below which only the pylorus was visible. The stomach was brought down into the abdomen, and an anterior gastropexy performed. He was discharged 12 days later and is well at six month follow-up.

There are few reported cases of gastrointestinal causes of extrapericardial cardiac compression producing haemodynamic compromise. We believe this is the second report involving a gastric volvulus. The echocardiographic features of diaphragmatic herniae have been previously characterised, with the hiatus hernia usually being visualised as an amorphous solid mass compressing the atria. We postulate that, in our case, there was significant compression of both atria causing tamponade. Although the patient suffered an EMD cardiac arrest, the physical act of cardiac massage may have resulted in partial decompression of the volvulus, since the haemodynamic parameters were much improved immediately postarrest.

P R KALRA R FRYMANN

D R ALLEN 